\title{
Cardioneuroablação: A Denervação Vagal por Cateter Como Nova Terapia para Síncope Cardioinibitória
}

\author{
Cardioneuroablation: Catheter Vagal Denervation as a New Therapy for \\ Cardioinhibitory Syncope
}

José Carlos Pachón Mateos ${ }^{1,2,3,{ }^{*}}$, Enrique Indalécio Pachón Mateo ${ }^{1,2}$, Christian Higuti 1,2, Tasso Júlio Lobo², Tomas Guillermo Santillana Peña², Carlos Thiene Cunha Pachón², Juan Carlos Pachón Mateos $^{1,2,3}$, Juán Carlos Zerpa Acosta2 ${ }^{2}$, Felipe Ortencio², Ricardo Amarante ${ }^{1,2}$.

ORCID IDS

Mateos JCP D https://orcid.org/0000-0002-5111-488X

Mateos EIP (D) https://orcid.org/0000-0001-7509-0731

Higuti C (D) https://orcid.org/0000-0003-3820-8600

Lobo TJ (D) https://orcid.org/0000-0002-2801-1903

Peña TGS (D) https://orcid.org/0000-0001-5795-9827

\author{
Pachón CTC Didhttps://orcid.org/0000-0003-3943-4722 \\ Mateos JCP (D) https://orcid.org/0000-0003-4410-8753 \\ Acosta JCZ (D) https://orcid.org/0000-0001-7436-2299 \\ Ortencio F (D) https://orcid.org/0000-0003-1805-0420 \\ Amarante R (D) https://orcid.org/0000-0002-7181-8198
}

\begin{abstract}
RESUMO
A síncope vasovagal é a causa mais frequente de perda transitória de consciência, especialmente em jovens sem doença cardíaca significativa. A forma cardioinibitória maligna é causada por reflexo vagal abrupto e intenso com ou sem gatilhos definidos. Casos refratários a medidas preventivas e manuseio farmacológico foram tratados com implante definitivo de marcapasso. Além de apresentar resultados questionáveis, o implante de marcapasso é altamente rejeitado por pacientes jovens. No final dos anos 1990, propusemos uma denervação vagal específica por ablação do cateter e mapeamento espectral para FA paroxística, bradiarritmias funcionais e casos graves de síncope cardioinibitória maligna dando origem à cardioneuroablação. Recentemente, muitos autores em todo o mundo vêm reproduzindo os resultados da cardioneuroablação, onde se observou eliminação ou redução significativa da resposta vagal, o que aboliu sintomas em mais de $75 \%$ dos pacientes acompanhados por até 14 anos, sem complicações. Portanto a cardioneuroablação tem se mostrado uma verdadeira opção terapêutica na síncope cardioinibitória maligna e em qualquer bradiarritmia vagal exclusiva mediada sem a necessidade de implante de marcapasso.
\end{abstract}

PALAVRAS-CHAVE: Síncope Vasovagal; Síncope Neurocardiogênica; Ablação por cateter; Estimulação do Nervo Vago.

\begin{abstract}
The vasovagal syncope is the most frequent cause of transient loss of consciousness, especially in young people without significant heart disease. Malignant cardioinhibitory form is caused by abrupt and intense vagal reflex with or without defined triggers. Refractory cases to preventive measures and pharmacological handling, has been treated with definitive pacemaker implantation. Besides showing questionable results, pacemaker implantation is highly rejected by young patients. In the late 1990s, we proposed a specific vagal denervation by catheter ablation and spectral mapping, for paroxysmal AF, functional bradyarrhythmias and severe cases of malignant cardioinhibitory syncope giving rise to cardioneuroablation. Recently, many authors worldwide have been reproducing the cardioneuroablation results where elimination or significant reduction of the vagal response were observed, which abolished symptoms in more than $75 \%$ of patients followed up to 14 years, without complications. Therefore, cardioneuroablation has shown to be a real therapeutic option in malignant syncope cardioinhibitory and in any exclusive vagal mediated bradyarrhythmia without the need of pacemaker implantation.
\end{abstract}

KEYWORDS: Vasovagal Syncope; Malignant Neurocardiogenic Syncope; Catheter Ablation; Vagus Nerve Stimulation.

1.Universidade de São Paulo - São Paulo (SP) - Brasil.

2. Hospital do Coração - Serviço de Eletrofisiologia, Marcapasso e Arritmias - São Paulo (SP) - Brasil.

3.Instituto Dante Pazzanese de Cardiologia - Serviço de Marcapasso - São Paulo (SP) - Brasil.

*Autor correspondente: pachon@usp.br

Recebido: 08 Nov 2019 | Aceito: 19 Nov 2019

Editor Associado: J. Tarcísio Medeiros de Vasconcelos 


\section{INTRODUÇÃO}

De acordo com o Consenso dos Especialistas da Heart Rhythm Society em 2015, síncope é definida como uma perda transitória de consciência, associada a uma incapacidade de manter o tom postural, a recuperação rápida e espontânea, e a ausência de características clínicas específicas para outra forma de perda transitória de consciência, como a convulsão epiléptica ${ }^{1}$. As causas comuns de síncope são a hipotensão vasovagal e a ortostática e distúrbios cardíacos. A síncope vasovagal, neurocardiogênica ou reflexa é a mais frequente, especialmente na população mais jovem, sem doença cardíaca aparente ${ }^{1}$. É um problema clínico muito frequente que reduz a qualidade da vida e pode aumentar o risco de trauma. Normalmente é uma perda transitória de consciência devido a uma resposta reflexa, causada por bradicardia intensa ou assistolia (tipo cardioinibitório) associada a maior ou menor grau de vasodepressão (tipo misto). A síncope vasovagal é raramente causada por vasodepressão isolada (tipo vasodepressor).

Apesar dos resultados controversos e de algum grau de incerteza, o marcapasso tem sido considerado o tratamento de escolha para síncope cardioinibitória grave sem resposta ao tratamento clínico ${ }^{1,2}$. Entretanto, além de apresentar um resultado questionável, o marcapasso está associado a grande rejeição por parte destes pacientes, geralmente jovens e sem doença cardíaca aparente. Como a resposta cardioinibitória é mediada por um enorme reflexo vagal, a primeira proposta de ablação autonômica, mais especificamente a denervação vagal através do uso da ablação por cateter de radiofrequência (RF) nas paredes atriais (cardioneuroablação - CNA) foi publicada em 2005, como tratamento definitivo para a síncope cardioinibitória grave, sendo o primeiro paciente ablacionado em $2002^{1}$. A grande vantagem desse método foi a possibilidade de tratamento sem implante de marcapasso.

\section{Síncope vasovagal}

Também conhecida como síncope reflexa, neurocardiogênica ou vasovagal, é causada por um mecanismo reflexo, através de múltiplos gatilhos, terminando com intensa cardioinibição e/ou vasodilatação levando à hipotensão severa e transitória. A cardioinibição pode ser suficiente para causar assistolia prolongada. Tipicamente, o paciente recupera-se espontaneamente em questão de minutos. Dependendo da fisiopatologia, pode ser classificada como cardioinibitória, vasodepressora ou mista. A primeira é caracterizada por assistolia, bradicardia grave e/ou bloqueio $\mathrm{AV}$ total transitório causado por intensa ação vagal. $\mathrm{Na}$ forma vasodepressora, geralmente ocorre hipotensão grave sem bradicardia significativa, principalmente devido a uma súbita redução do tom simpático ${ }^{2}$. Na forma mista, há a contribuição mais ou menos intensa dos dois mecanismos.

\section{SISTEMA NERVOSO AUTÔNOMO CARDÍACO}

\section{Anatomia}

O sistema nervoso autônomo do coração consiste em três grandes divisões, uma aferente - o sistema nervoso sensorial -e duas partições eferentes, os ramos parassimpático e simpático. A medula oblonga é o principal centro de integração da inervação cardíaca, cuja atividade é modulada pelo hipotálamo e por centros mais superiores. As fibras sensoriais são neurônios bipolares cujos corpos celulares estão na medula oblonga. As fibras eferentes compreendem essencialmente dois neurônios principais, pré e pós-ganglionares. A fibra parassimpática pós-ganglionar é muito curta porque seu corpo neuronal está localizado no coração, principalmente na parede atrial e nos plexos ganglionados (placas de gordura paracárdica) ${ }^{3}$. O corpo celular do neurônio pré-ganglionar parassimpático está situado na medula oblonga, mais especificamente no núcleo ambíguo e no núcleo motor dorsal do vago ${ }^{4}$. Seu axônio é levado ao coração pelos nervos vagos. Em contraste, o neurônio pós-ganglionar simpático é muito longo porque seu corpo celular está localizado na cadeia paravertebral simpática. O resultado dessa distribuição é que apenas o corpo neuronal pós-ganglionar parassimpático está localizado no coração e apenas ele é propenso a ser eliminado pela ablação endocárdica por RF.

\section{Fisiologia}

Apesar de o coração ser um músculo estriado, sua atividade não depende da inervação. Ao contrário do músculo esquelético que atrofia quando denervado, o coração retém seu metabolismo, sua estrutura e sua atividade normais independentemente da inervação. Esse fato é facilmente observado em pacientes com transplante cardíaco. Entretanto o sistema nervoso autônomo atua permanentemente modulando todas as propriedades cardíacas, através de um intenso tônus inibitório (parassimpático) e excitatório (simpático). Esse 
antagonismo funcional cria um equilíbrio mediado pelo tronco cerebral que determina o ritmo cardíaco instantâneo. Este equilíbrio é constantemente restaurado pelo sistema nervoso autônomo, ajustando a fisiologia cardíaca a cada momento. Assim, no caso da denervação vagal, uma redução proporcional do tônus simpático determinada pelo equilíbrio reflexo do sistema nervoso autônomo é normalmente observada, trazendo a frequência cardíaca de volta à linha de base.

\section{Fundamentação}

A cardioinibição da síncope reflexa é causada por um reflexo vagal súbito maciço que pode ser totalmente eliminado pela denervação vagal. Isso também é elegantemente comprovado pela prevenção da síncope cardioinibitória por atropina ${ }^{5}$. Visando um novo tratamento definitivo para essa condição, no final dos anos 1990, propusemos uma denervação vagal específica ${ }^{5}$ por ablação endocárdica guiada por mapeamento espectral, para tratamento de fibrilação atrial (FA) paroxística, bradiarritmias funcionais e síncope cardioinibitória grave, dando origem à cardioneuroablação. Desde então, temos estudado a possibilidade de abolir ou atenuar permanentemente o reflexo cardioinibitório, utilizando a ablação endocárdica por RF. Embora uma denervação temporária possa ser obtida com a eliminação das fibras neurais, a denervação a longo prazo necessita da eliminação dos corpos celulares dos neurônios para evitar a reinervação. Como mencionado anteriormente, a inervação cardíaca é composta por sistemas parassimpáticos, simpáticos e sensoriais (Fig. 1). Apenas a inervação parassimpática contém a maior parte do neurônio pós-ganglionar dentro do coração, mais especificamente dentro da parede atrial, no epicárdio, no endocárdio ou nos gânglios paracárdicos (placas de gordura epicárdica). Em contraste, os corpos celulares dos neurônios pós-ganglionares simpáticos e sensoriais encontram-se, respectivamente, nos gânglios da cadeia paravertebral e no sistema nervoso central, longe do coração. Essas características favorecem uma denervação vagal mais seletiva, uma vez que o corpo celular do neurônio pós-ganglionar parassimpático é o único exposto diretamente à aplicação de RF nas paredes atriais. Os neurônios simpáticos e sensoriais do corpo são preservados e sua função é normalmente recuperada porque apenas as terminações das fibras são temporariamente eliminadas pela energia de RF no endocárdio ${ }^{6,7}$.

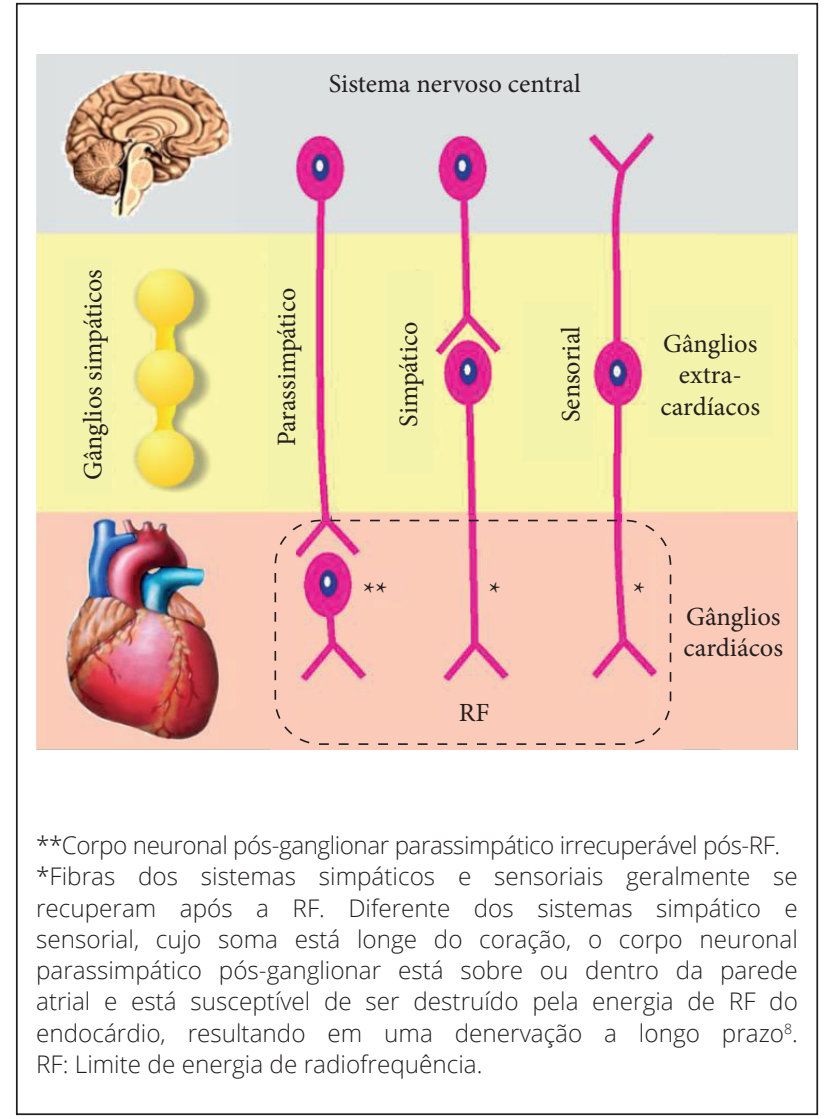

Figura 1. Esboço do sistema nervoso cardíaco com as três principais partições da inervação cardíaca e o efeito da RF endocárdica.

\section{DENERVAÇÃO CARDÍACA AUTONÔMICA}

Foram desenvolvidas várias tentativas de denervação cardíaca com outros fins que não o tratamento de cardioinibição. A estelectomia esquerda para o tratamento da síndrome do QT longo é um exemplo de sucesso ao ablacionar parcialmente o sistema nervoso simpático do coração ${ }^{9}$. Mais recentemente, a ablação cirúrgica dos plexos ganglionados (PGs) epicárdicos visando o tratamento da fibrilação atrial tem sido tentada ${ }^{10,11}$. Infelizmente, esse tipo de denervação não mostrou resultados positivos ${ }^{12}$. Em contraste, a denervação vagal para tratar a síncope vasovagal parece ser altamente eficiente. $O$ primeiro estudo propondo esse tipo de denervação por ablação endocárdica para eliminar a cardioinibição causada pelo reflexo vagal foi publicado em $2005^{5}$, e o primeiro paciente foi tratado em 2002. Desde então, foram obtidos avanços significativos e muitos autores distintos conseguiram reproduzir os resultados ${ }^{13-21}$. 


\section{Ablação por RF via cateter para denervação vagal}

O principal objetivo do procedimento é o mapeamento e a ablação das terminações vagais na parede atrial. Como o neurônio pós-ganglionar parassimpático está localizado dentro ou sobre a parede atrial e nos plexos ganglionados cardíacos, há uma grande chance de conseguir sua eliminação por RF convencional do endocárdio. No entanto o principal desafio é a detecção e o mapeamento de áreas de inervação endocárdica de alta densidade, a fim de liberar a RF. A hipótese era que a RF endocárdica poderia ser capaz de eliminar o neurônio pós-ganglionar parassimpático existente na parede atrial (primeiro neurônio, Fig. 2), no epicárdio visceral e até parte dos neurônios localizados nos plexos ganglionados (segundo neurônio, Fig. 2). O objetivo era obter denervação suficiente para eliminar ou atenuar o tônus vagal e a cardioinibição a longo prazo, tornando o reflexo cardioinibitório vasovagal mais resistente a gatilhos.

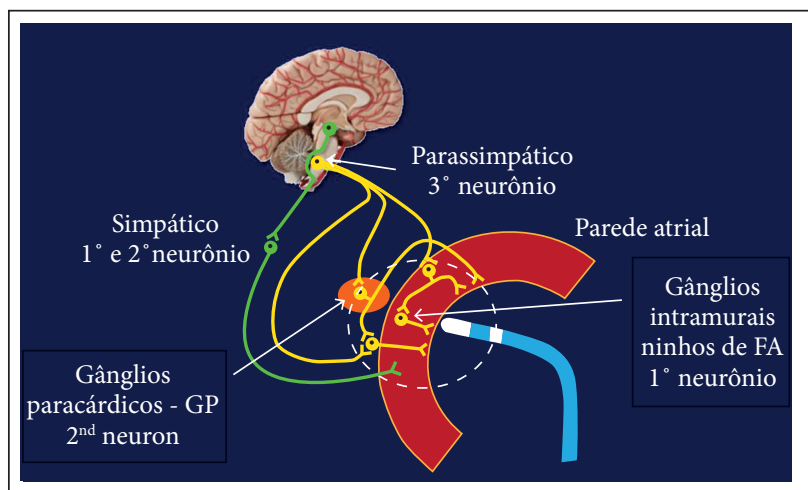

Nota: Existem numerosos grupos de neurônios pós-ganglionares parassimpáticos na parede atrial (ninhos de FA - primeiro neurônio) e vários gânglios no epicárdio dentro das placas de gordura (plexos ganglionados - segundo neurônio). O terceiro neurônio está localizado no centro nervoso no núcleo dorsal do nervo vago, nos núcleos solitário e ambíguo. A energia de RF pode eliminar a maior parte do primeiro e do segundo neurônios, já que a profundidade de aquecimento alcançada com cateteres irrigados pode ser de até $1,5 \mathrm{~cm}$, possível borda de aquecimento.

Figura 2. Esquema da inervação cardíaca e da cardioneuroablação.

\section{Mapeamento e ablação da inervação cardíaca por abordagem endocárdica}

Ao contrário do músculo esquelético, o miocárdio não tem uma junção neuromuscular diferenciada. Em contraste, as fibras parassimpáticas e simpáticas do nervo penetram diretamente no miocárdio e se entrelaçam com os miócitos. Adicionalmente, muitos microneurônios (neurônios pós-ganglionares parassimpáticos, primeiro neurônio na Fig.2) colonizam as paredes atriais (Fig. 4 e 5). Essa mistura de células altera algumas propriedades elétricas da parede atrial e pode ser identificada por alterações no espectro como "ninhos de FA"22-25 (esse nome é resultado da sua relação com a fisiopatologia da FA, pois apresentam ressonância elétrica ${ }^{26,27}$ favorecendo a manutenção da FA). Portanto, usando o estudo espectral durante o ritmo sinusal, encontram-se dois tipos de miocárdio. O primeiro, o miocárdio compacto, caracterizado por alta amplitude, condução isotrópica e um espectro suave (Fig. 3a), enquanto o segundo, o miocárdio fibrilar, tem baixa amplitude, condução anisotrópica e espectro segmentado (Fig. 3b) $)^{26,28,31}$.

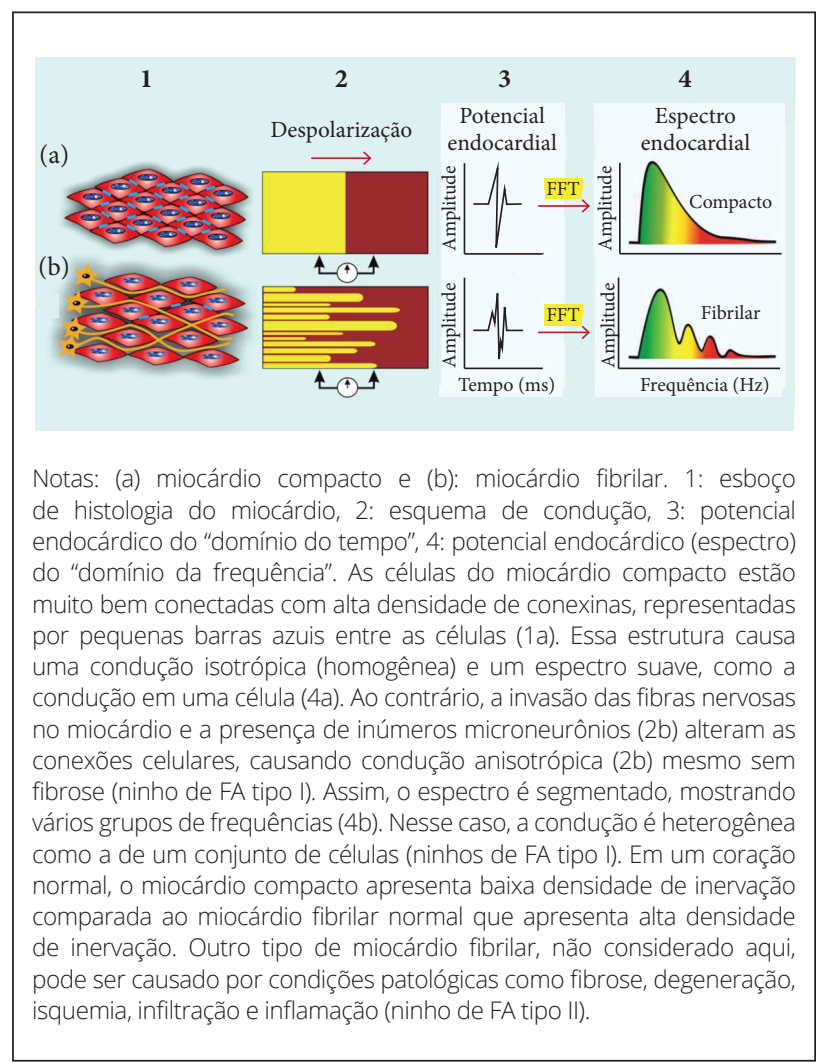

Figura 3. Diferenças nas propriedades eléctricas do miocárdio, dependendo do grau de ligação das células.

Aglomerados de miocárdio fibrilar dão origem a ninhos de FA. Vários resultados têm demonstrado uma estreita relação entre o miocárdio fibrilar e a interface de inervação cardíaca:

- O fornecimento de RF no miocárdio septal fibrilar geralmente causa reações autonômicas imediatas, tais como uma frequência cardíaca significativa e o aumento do ponto de Wenckebach ${ }^{8,29,30}$;

- Estudos detalhados baseados na coloração neural mostraram um número elevado de neurônios e 
gânglios parassimpáticos nas áreas do miocárdio fibrilar ${ }^{31-33}$;

- Uma grande quantidade de miocárdio fibrilar é encontrada nas regiões anatômicas dos plexos ganglionados cardíacos ${ }^{26,27,31}$.

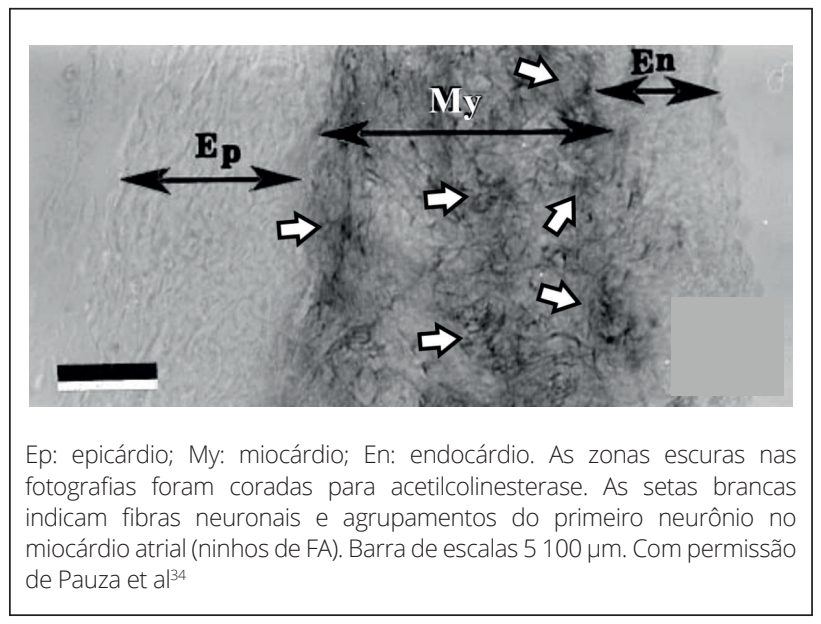

Figura 4. Fotomicrografias de cortes transversais da parede atrial direita de um cão adulto.

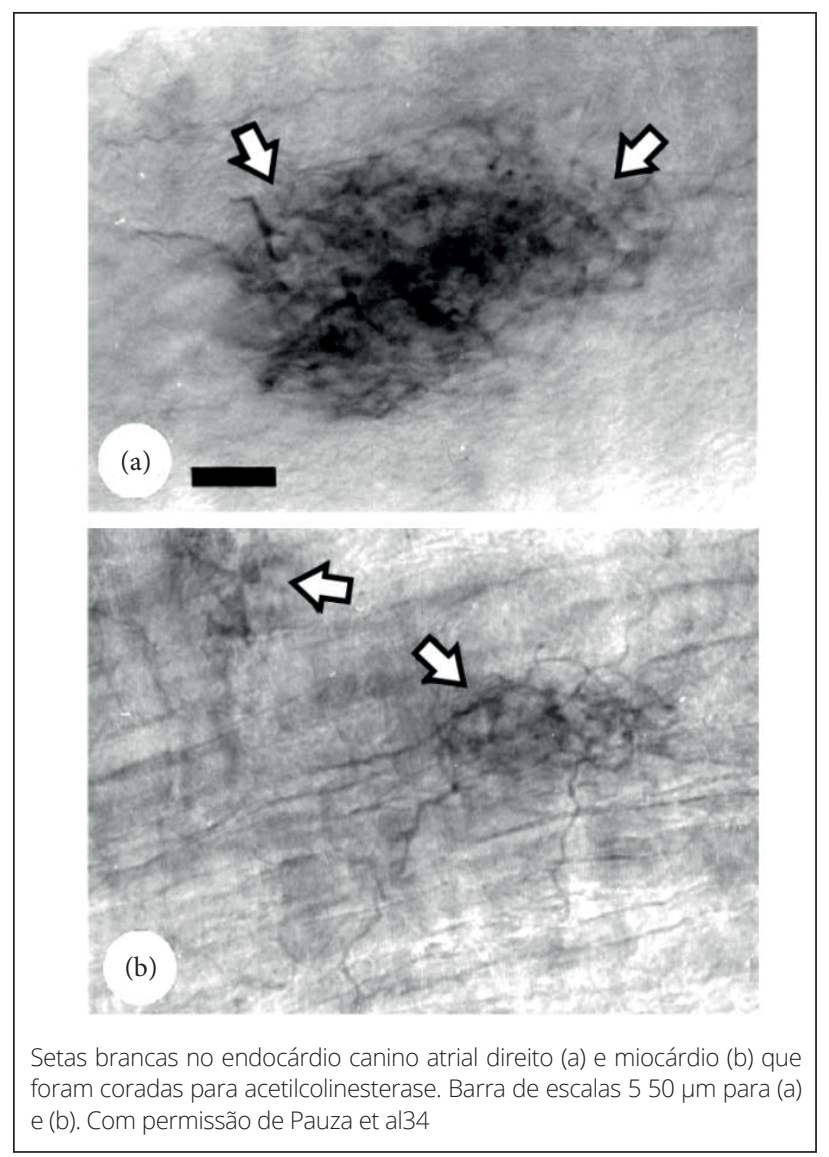

Figura 5. Exemplos de agrupamentos do primeiro neurônio na parede atrial (ninhos de FA).

Através do mapeamento espectral on-line em tempo real, é possível revelar o miocárdio fibrilar (ninhos de FA) para orientar a ablação (Fig. 3b) do primeiro neurônio.
Consequentemente, a maioria dos neurônios pós-ganglionares parassimpáticos pode ser abolida e não se recupera, enquanto as fibras terminais simpáticas e sensoriais geralmente se recuperam entre semanas e meses (Fig. 2). As fibras vagais pré-ganglionares podem fornecer algum grau de reinervação, mas são reduzidas porque perderam o elo pós-ganglionar substituído por alguma fibrose pós-RF.

\section{MÉTODODECARDIONEUROABLAÇÃO Critérios de inclusão}

O sucesso do procedimento depende de um critério rigoroso de inclusão. O principal é a presença de bradiarritmia reflexa e/ou funcional em um paciente sintomático, sem resposta ou sem possibilidade de tratamento clínico ${ }^{35} \mathrm{em}$ um coração aparentemente normal ou tendo excluído racionalmente uma cardiopatia significativa ${ }^{36}$. Da mesma forma, os testes farmacológicos para confirmar a reversibilidade da condição, como resposta positiva à atropina, são decisivos (Tabela 1).

Tabela 1. Critérios de inclusão da cardioneuroablação. Durante o teste de atropina, o ritmo cardíaco deve duplicar ou alcançar mais de $100 \mathrm{bpm}$ por pelo menos $15 \mathrm{~min}$.

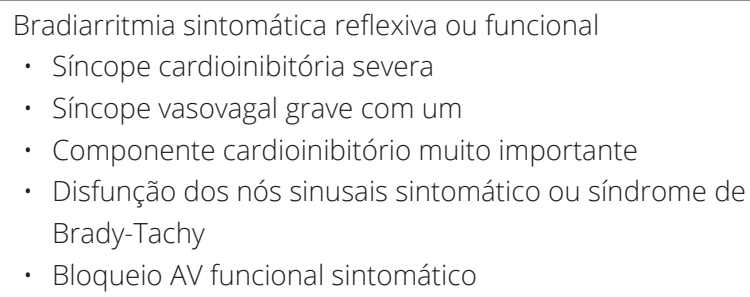

\section{MÉTODO}

Uma vez que o paciente assinou o termo de consentimento, o procedimento é realizado sob anestesia geral. Uma preocupação importante é que o anestesiologista possa inadvertidamente usar atropina no início do procedimento e isso faria com que os cardiologistas perdessem o parâmetro do tom autonômico; portanto é essencial alertar a equipe de anestesistas para discutir antes de usar qualquer medicamento autonômico. 
Os sinais vitais (frequência cardíaca, oximetria, pressão sanguínea, pletismografia, perfusão periférica, capnografia e gases respiratórios) são rigorosamente monitorizados. Adesivos de pele do 3D $\mathrm{NavX}^{\mathrm{TM}}$ ou o Sistema de Mapeamento Eletroanatômico Velocity ${ }^{\mathrm{TM}}$ são aplicados e o transdutor de ecocardiografia esofágica é inserido. A função cerebral é monitorada por espectral (BIS Aspect A-1000) mantendo o nível de consciência entre 40 e 50 índices biespectrais. São colocadas derivações eletrofisiológicas (EF) convencionais e um estudo EF de rotina é realizado. A punção do septo interatrial é realizada sob orientação de ecocardiograma transesofágico. A heparina (100 IU/kg) é utilizada para manter o tempo de coagulação ativado em torno de 300 s. O mapeamento é realizado com um espectrômetro ou através da configuração dos filtros dos gravadores EF convencionais.

\section{RF guiada por mapeamento espectral ou por potenciais endocárdicos}

O paciente deve estar em ritmo sinusal e os átrios direito e esquerdo são escaneados com cateter convencional irrigado com o gerador de RF termocontrolado. A RF é aplicada em todos os locais caracterizados como ninho de FA (miocárdio fibrilar) (Fig. 6) visando a eliminação do primeiro neurônio. A digitalização pode ser guiada por mapeamento espectral (Fig. 7) ou por gravações convencionais com algumas configurações de filtragem (Fig. 8).

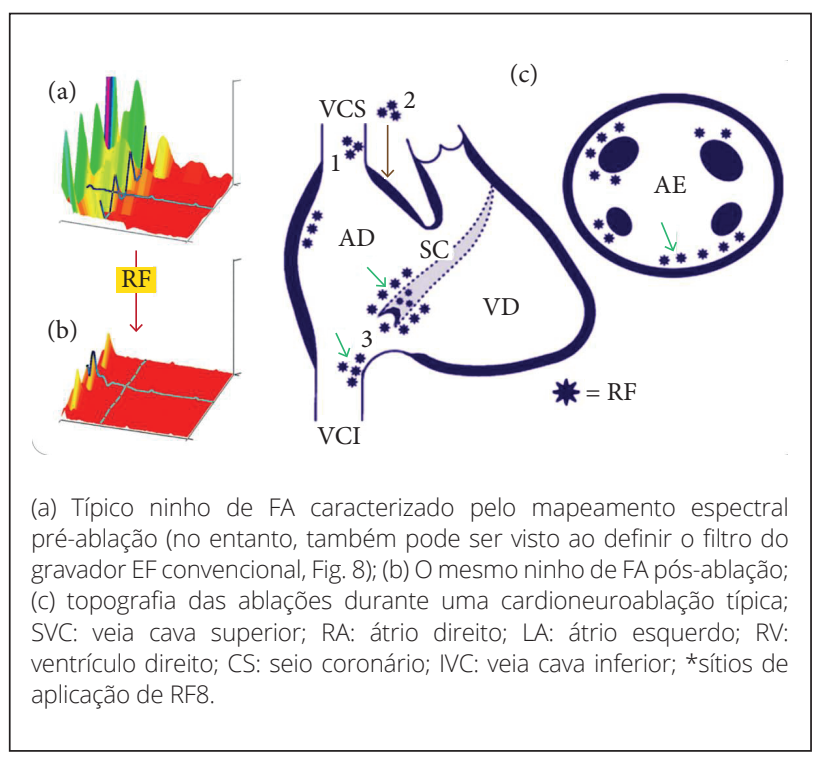

Figura 6. Locais principais do ninho de FA.

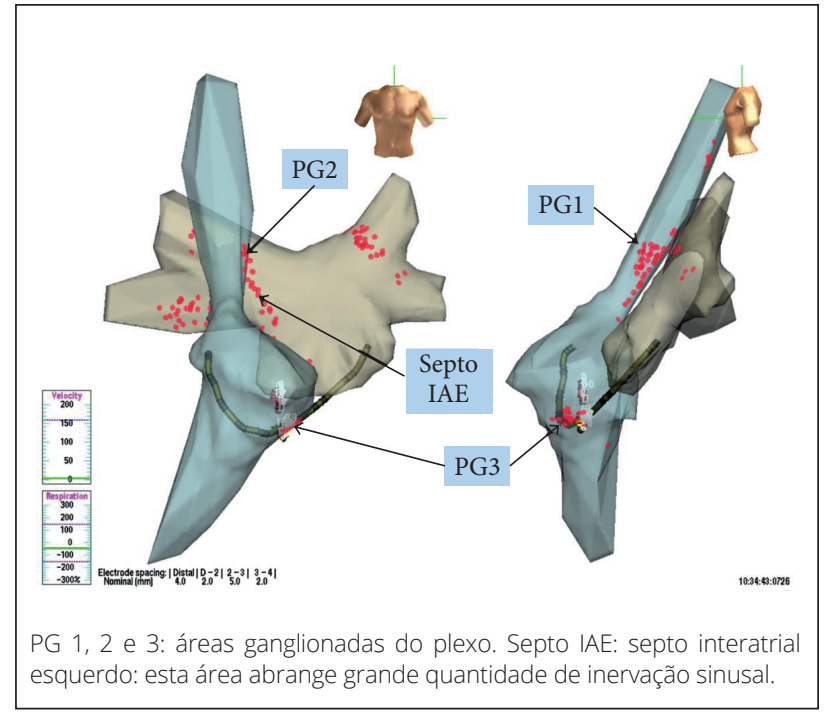

Figura 7. Mapeamento eletroanatômico típico no final de uma CNA regular (não editado).

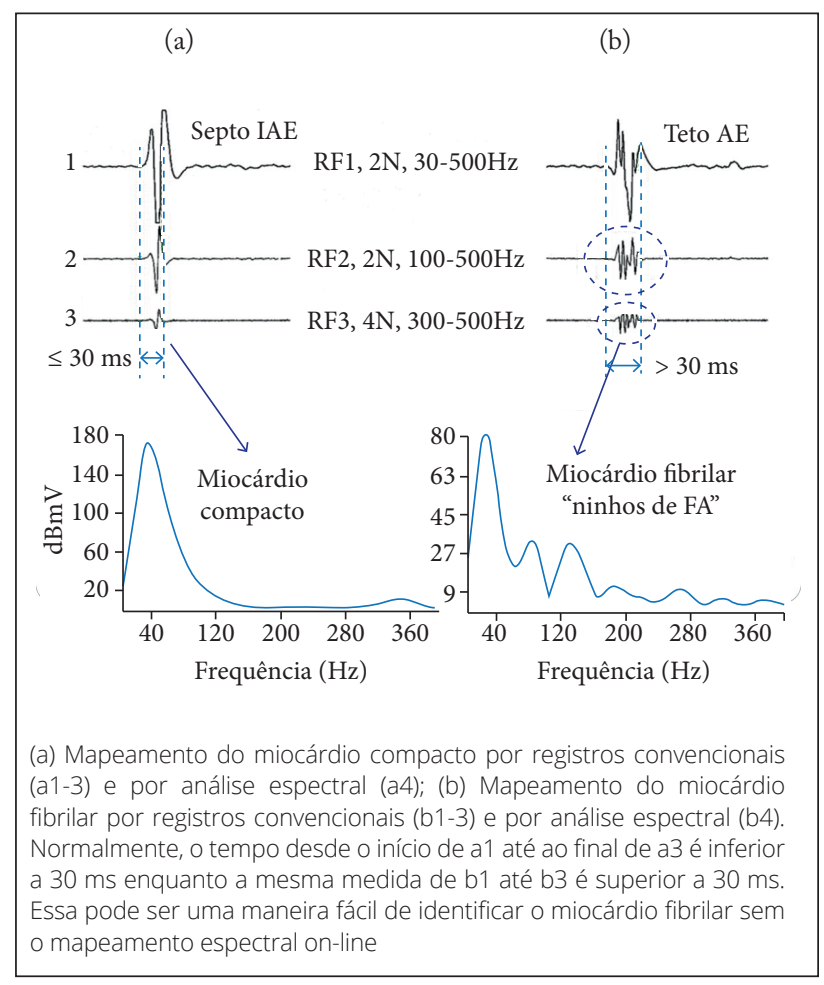

Figura 8. Registro convencional e espectral

\section{RF guiada por parâmetros anatômicos}

Após a ablação de todo o miocárdio fibrilar escaneado, também é realizada ablação anatômica endocárdica (pelo menos 2 min em cada local), nas áreas relacionadas aos plexos ganglionados (PGs) epicárdi$\cos ^{37}$. Essas áreas normalmente apresentam um elevado número de ninhos de FA e permitem a eliminação ou 
despovoamento do segundo neurônio. A RF nestas áreas deve ser estendida para obter aquecimento profundo:

1. PG1: Na veia cava superior, medial e logo acima da inserção atrial da veia cava (Figs. 6c, 7 e 9-1);

2. PG2: No septo interatrial esquerdo, entre as veias pulmonares direitas e a fossa oval (ablacionado através do AE) e da fossa oval até o sulco de Waterston (ablacionado através do AD) (Figs. 6c, 7 e 9);

3. PG3: $\mathrm{O}$ teto do seio coronário (ablacionado através do $\mathrm{AE}$ ) e pela veia cava inferior, medial e logo abaixo da valva tricúspide e do óstio do seio coronário (Figs. $6 c, 7$ e 9);

4. PGn: Existem vários outros $\mathrm{PGs}$ relacionados inclusive com a inserção da veia pulmonar ${ }^{34}$. A ablação auxiliar dessas fontes pode ser obtida em maior ou menor grau durante o isolamento das veias pulmonares (Fig. 7).

\section{Metodologia para controlar e confirmar a denervação vagal}

Neste procedimento, é absolutamente essencial ter a demonstração do efeito de inervação vagal, o controle gradual da denervação durante o procedimento e, finalmente, a confirmação da denervação vagal no final do procedimento, como um critério de sucesso imediato. Para isso, desenvolvemos a estimulação vagal extracardíaca, que é uma estimulação neural muito fácil de atender a todos esses passos. O procedimento começa com o avanço de um cateter eletrofisiológico dentro das veias jugulares internas direita ou esquerda até o nível do forame jugular (Figs. 10 e 11).

O estimulador vagal libera pulsos de onda quadrada de $50 \mathrm{~ms}$ de largura, frequência de $30 \mathrm{~Hz}$ e amplitude de 10 a $70 \mathrm{~V}$, ajustados de acordo com o peso do paciente $(1 \mathrm{~V} / \mathrm{kg}$ até $70 \mathrm{~V})$. Uma duração de pulso extremamente curta com limitação de corrente é empregada para prevenir a lesão tecidual. Uma função temporizada permite a aplicação de trens de pulso com temporização pré-definida, normalmente entre 5 a $10 \mathrm{~s}$.

Uma nova estimulação vagal é repetida no final da CNA e comparada com a primeira. O procedimento é concluído se a eliminação total da resposta vagal for confirmada (Fig. 12b). Estimulações vagais adicionais durante o procedimento são usadas para mostrar o progresso da denervação vagal.

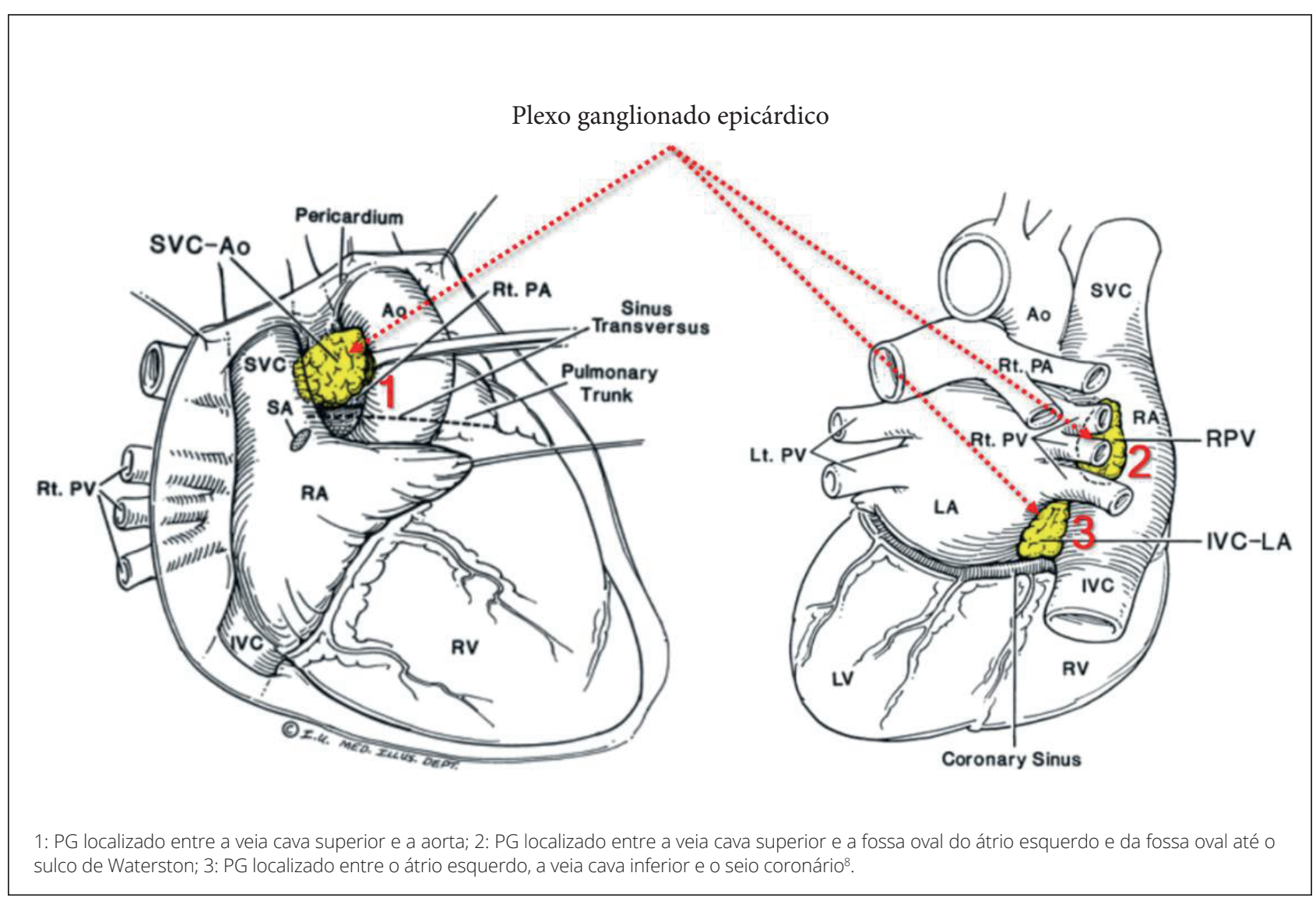

Figura 9. Topografia do principal plexo ganglionado (PG) epicárdico ${ }^{38}$. 


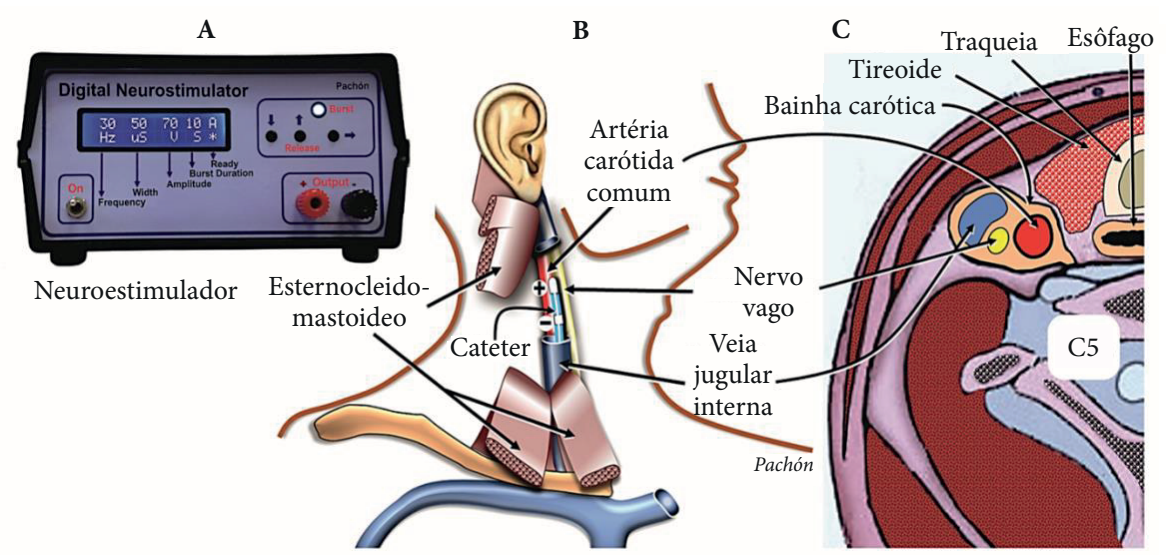

(a) neuroestimulador; (b) esquema da progressão do cateter EF dentro da veia jugular interna até o forame jugular; (c) secção transversal mostrando a estreita relação do vago com a jugular interna ${ }^{33}$.

Figura 10. Metodologia da estimulação vagal durante a cardioneuroablação.

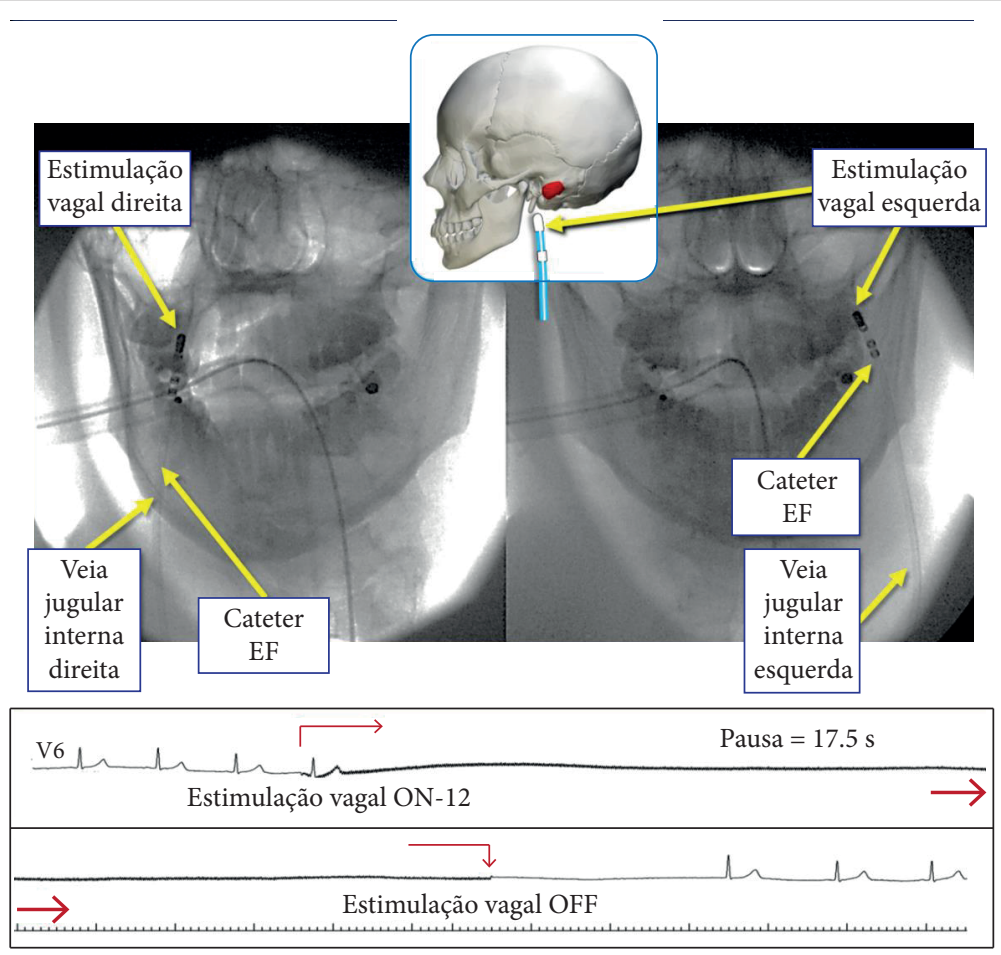

Raios-X mostrando a posição do cateter EF para estimulação vagal. Um cateter é avançado dentro da veia jugular interna até o forame jugular. Abaixo, um exemplo da estimulação vagal direita. Há uma assistolia longa que dura poucos segundos além da neuroestimulação, dependendo da recuperação do nó sinusal e do metabolismo da acetilcolina.

Figura 11. Sítio de estimulação vagal.

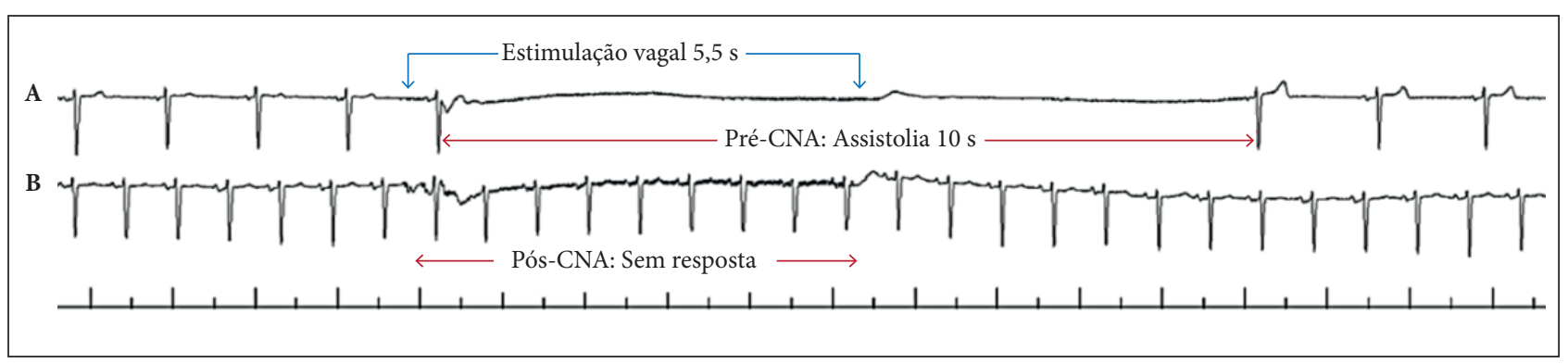

Figura 12. Estimulação vagal antes (a) e no final (b) da CNA demonstrando uma denervação vagal bem-sucedida (abolição completa da resposta vagal). 


\section{Desfechos imediatos de cardioneuroablação}

Vários desfechos devem ser considerados para melhorar os resultados da CNA, como mostrado na Tabela 2 .

Tabela 2. Principais desfechos imediatos de cardioneuroablação.

Ablação da maioria dos ninhos de FA no átrio esquerdo e átrio direito

Ablação anatômica das principais áreas do plexos ganglionados

Aumento sustentado do ritmo cardíaco e do ponto de Wenckebach

Supressão completa da resposta vagal por estimulação vagal Abolição total da resposta atropínica

\section{Proteção esofágica durante a cardioneuroablação}

Quanto maior a ablação dos ninhos de FA, maior e mais duradoura será a denervação vagal. Além das regiões dos PGs, grande inervação vagal entra nos átrios pela inserção de veias pulmonares. Portanto é altamente desejável eliminar os ninhos de FA relacionados com essas áreas, como a ablação por fibrilação atrial. Nesse sentido, é necessário um cuidado especial com a proteção do esôfago. Para tanto, desenvolvemos um método, aplicado a todos os pacientes incluídos neste estudo, que é o deslocamento mecânico do esôfago através do transdutor ecocardiográfico transesofágico ${ }^{39}$. Normalmente, o deslocamento é suficiente para mover o esôfago de 4 a $8 \mathrm{~cm}$ na direção oposta ao ponto de RF, reduzindo expressivamente o risco de aquecimento e lesão do esôfago (Fig. 13).

\section{RESULTADOS DA \\ CARDIONEUROABLAÇÃO}

O resultado imediato no final da CNA é a ausência completa de resposta vagal indicando uma denervação vagal bem-sucedida (Fig. 12b). De fato, esse é o principal critério de sucesso e, se não for alcançado, a ablação deve ser retomada e ampliada até obter a eliminação completa da resposta vagal.

O resultado a longo prazo deve ser avaliado principalmente pelo acompanhamento clínico, mas é necessário realizar testes com a mesma avaliação que demonstrou a resposta cardioinibitória mais importante no momento da inclusão, mais frequentemente, o teste de inclinação (Fig. 14). No entanto, especialmente em casos de bradiarritmias funcionais não reflexivas, a cardioinibição pode ter sido detectada por um teste de esforço ou registro de Holter. O teste de inclinação é, sem dúvida, o teste mais usado como critério de inclusão e controle. Recomenda-se que seja repetido a partir de 2 meses pós-CNA usando o mesmo protocolo da fase de inclusão (Fig. 15).

Em um estudo de coorte de longo prazo com 43 pacientes cardioinibitórios, com seguimento de $45,1 \pm 22-11$ a 91,4 meses $^{41}$, foi observado controle positivo no teste de inclinação apenas em 4 casos (9,3\%), com o mesmo grau de cardioinibição mas, todos eles, com resposta vasodepressora significativa (Tabela 3 ).

Esses resultados mostram que o baixo número de pacientes que apresentam síncope após a CNA parece mudar o comportamento vasovagal da cardioinibição grave para uma resposta vasodepressora predominante menos importante, já que o procedimento diminui significativamente a resposta de cardioinibição, mesmo a longo prazo.
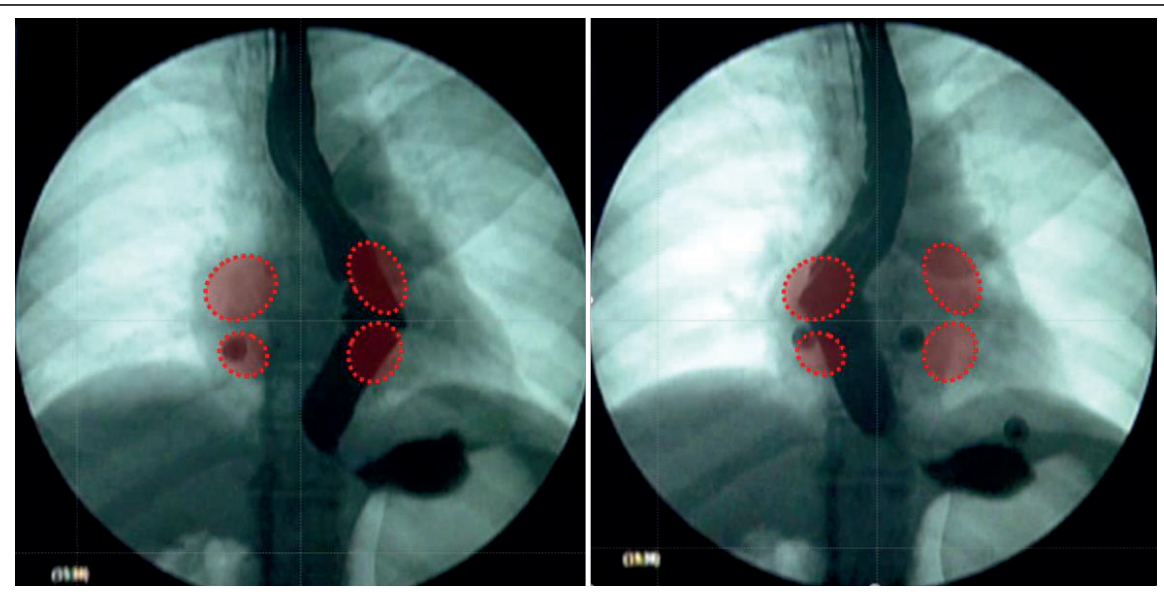

Figura 13. Radiografia contrastada do esôfago mostrando o deslocamento durante a ablação por RF no antro das veias pulmonares (círculos vermelhos). A extensão do deslocamento é suficiente para permitir uma boa proteção do esôfago na maioria dos pacientes ${ }^{40}$. 


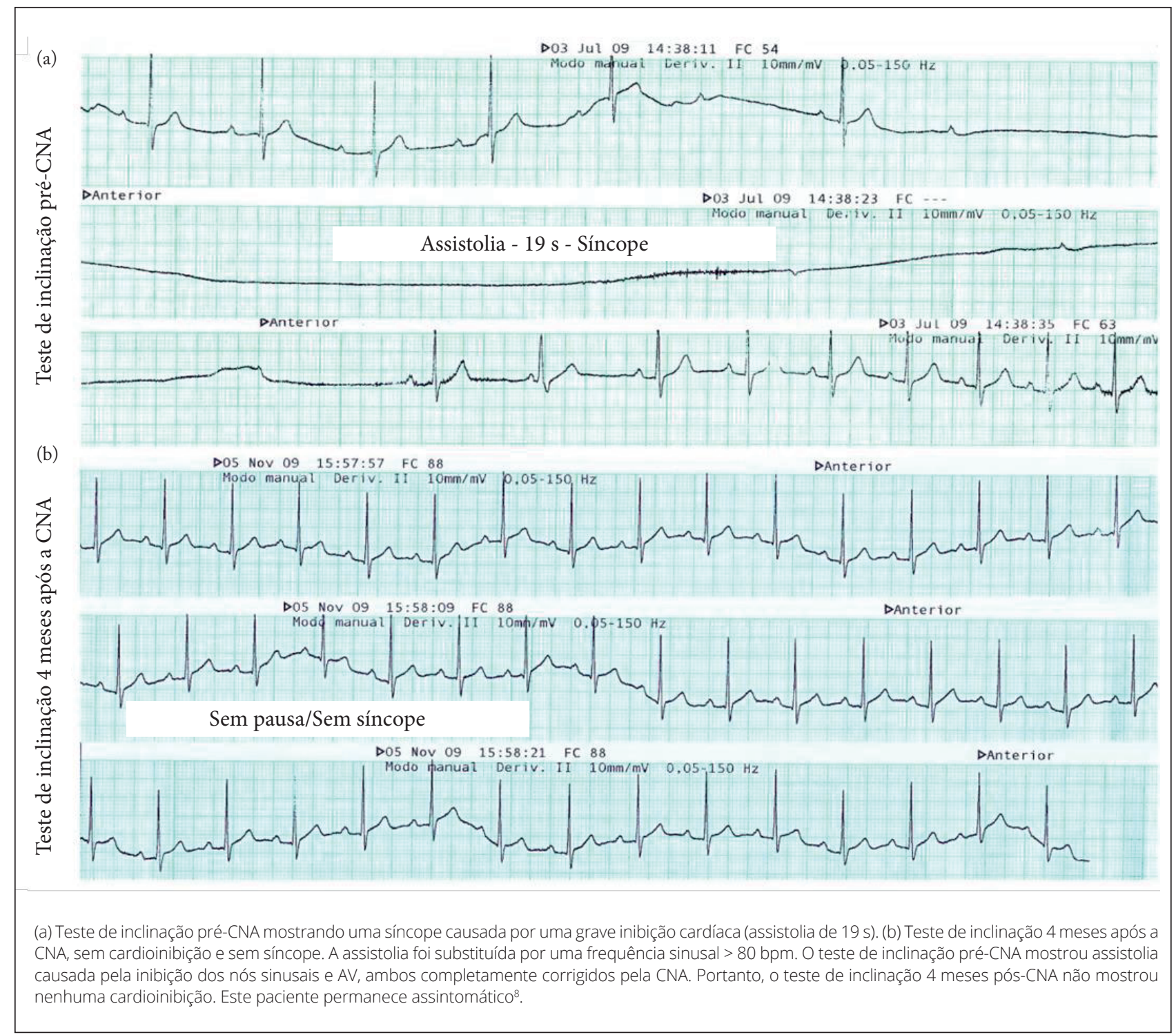

Figura 14. Teste de inclinação para obter o resultado da CNA a longo prazo.

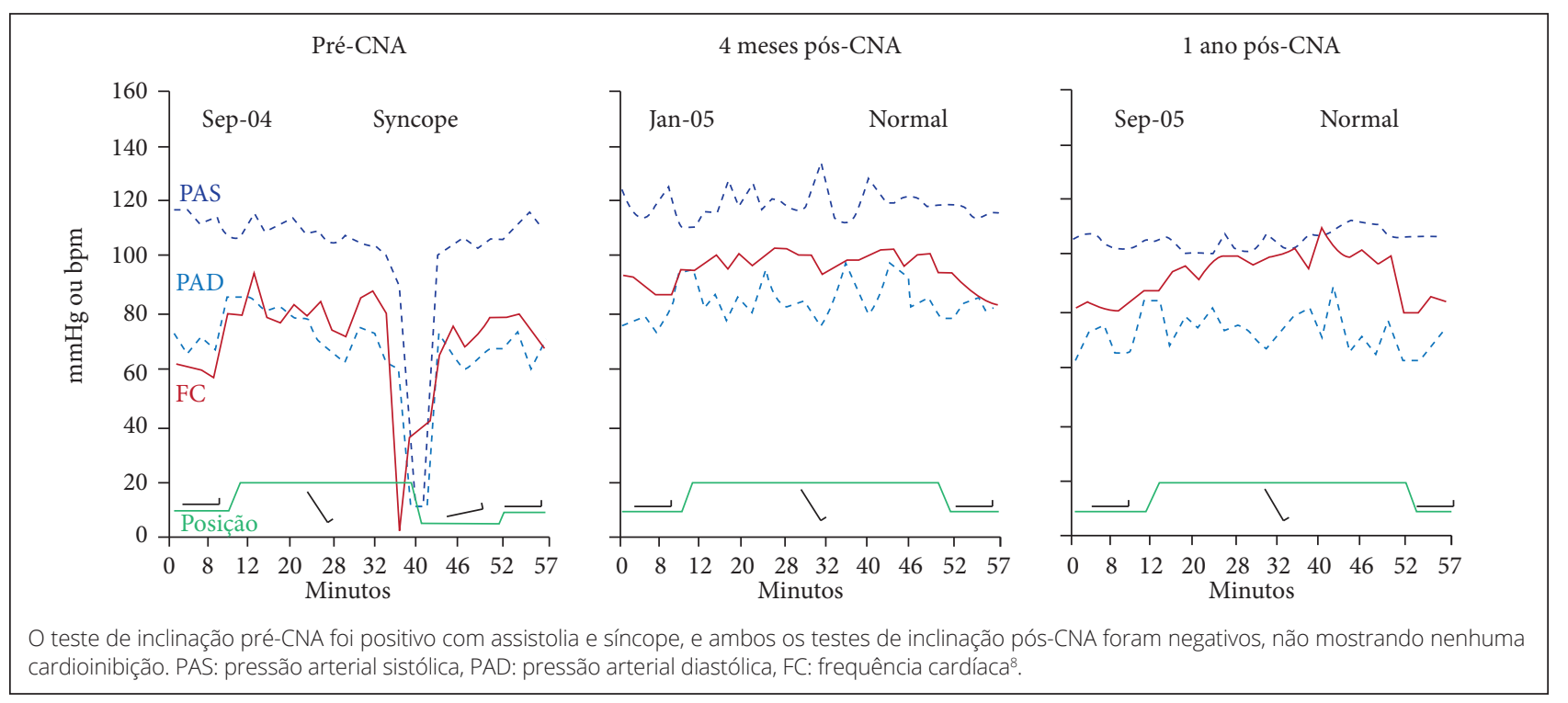

Figura 15. Teste de inclinação no seguimento a longo prazo da CNA. 
Tabela 3. Características dos casos com teste de inclinação positivo pós-CNA em uma coorte de 43 pacientes.

\begin{tabular}{|c|c|c|c|c|c|c|c|c|c|c|}
\hline \multirow[b]{2}{*}{ NIP } & \multirow[b]{2}{*}{ Idade } & \multicolumn{4}{|c|}{ Teste de inclinação pré-CNA } & \multicolumn{4}{|c|}{ Teste de inclinação pós-CNA } & \multirow[b]{2}{*}{ Resultado } \\
\hline & & MTI & Resposta & Cardioinhibição & $\begin{array}{c}\text { Pausa } \\
\text { (seg) }\end{array}$ & MTI & Resposta & $\begin{array}{l}\text { Cardio- } \\
\text { inibição }\end{array}$ & SE & \\
\hline 7 & 48 & N & Positivo/Misto & BJ & 3,7 & $\mathrm{~N}$ & $\begin{array}{l}\text { Positivo/ } \\
\text { Misto }\end{array}$ & BS & 75 & Assintomático \\
\hline 17 & 17 & N & $\begin{array}{c}\text { Positivo/ } \\
\text { Cardioinibitório }\end{array}$ & Assistolia & 12,0 & S & $\begin{array}{l}\text { Positivo/ } \\
\text { Misto }\end{array}$ & BS & 55 & $\begin{array}{c}\text { Tonturas (cólicas } \\
\text { menstruais) }\end{array}$ \\
\hline 26 & 14 & $\mathrm{~N}$ & Positivo/Misto & Pausa sinusal & 3,0 & $S(42)$ & $\begin{array}{l}\text { Positivo/ } \\
\text { Misto }\end{array}$ & BS & 43 & Assintomático \\
\hline 37 & 51 & S & $\begin{array}{c}\text { Positivo/ } \\
\text { Cardioinibitório }\end{array}$ & Assistolia & 6,0 & S & $\begin{array}{l}\text { Positivo/ } \\
\text { Misto }\end{array}$ & BJ & 17 & Assintomático \\
\hline
\end{tabular}

NIP: número de identificação do paciente; MTI: Medicamento do teste de inclinação; SE: seguimento em meses; BS: Bradicardia Sinusal; BJ: Bradicardia Juncional; Em todos os casos, foi observada uma redução significativa da cardioinibição em relação ao teste de inclinação pré-CNA ${ }^{8}$

\section{Curvas de sobrevivência da cardioneuroablação}

$\mathrm{O}$ valor terapêutico da $\mathrm{CNA}^{8}$ pode ser avaliado comparando-se os resultados com o tratamento clínico $^{42} \mathrm{e}$ com o implante do marcapasso nos estudos de ISSUE-3 ${ }^{3}$ e SYNPACE ${ }^{37}$. Na Fig. 16, mesmo considerando um seguimento mais longo do que outros estudos, utilizando-se o CNA apenas 6 dos 75 pacientes tinham síncope indeterminada

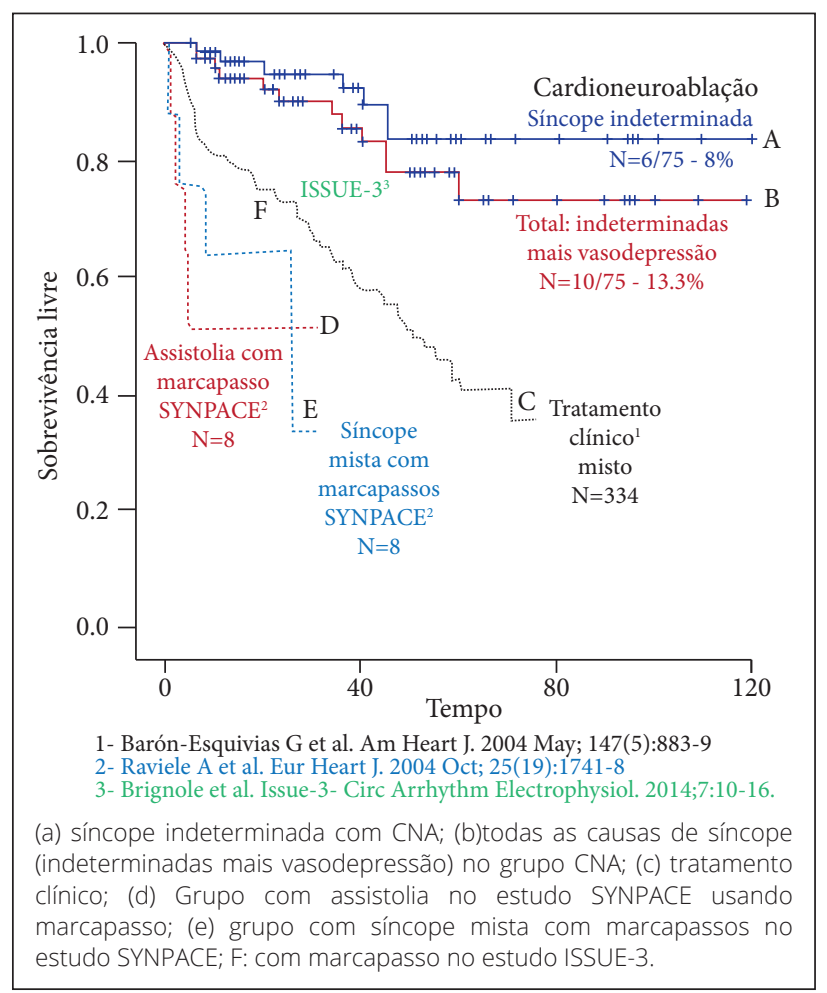

Figura 16. Curvas de sobrevivência livre de síncope comparando a CNA com o tratamento clínico e com o implante de marcapasso nos estudos ISSUE-3 e SYNPACE.
(8\%) e o total de casos com síncope indeterminada mais vasodepressor foi de 10, totalizando $13,3 \%$ em um seguimento médio de $38 \pm 28$ meses. Entretanto, se um período mais curto de 24 meses de acompanhamento for considerado, como no "Estudo Internacional de Síncope de Etiologia Incerta 3" (ISSUE 3), a incidência de síncope foi de $9 \%$ com CNA, 25\% com marcapasso no estudo ISSUE 3, 28\% com tratamento clínico, 35\% no grupo de síncope mista do estudo Synpace e 48\% no grupo assistólico do mesmo estudo, ambos com marcapassos. Assim, pode-se concluir que a CNA em pacientes com síncope vasovagal com importante componente cardioinibitório apresenta melhor resultado quando comparado ao tratamento clínico e implante de marcapasso, proporcionando ao médico uma nova opção de tratamento de casos difíceis.

\section{A CNA pode trazer consequências indesejáveis?}

O acompanhamento rigoroso dos nossos pacientes ao longo de 14 anos não mostrou nenhum efeito indesejável, mesmo nos poucos casos em que o procedimento foi refeito.

$\mathrm{Na}$ fase inicial, há uma mudança notável no tom autonômico comumente observado como taquicardia sinusal persistente. Por causa disso, é costume manter o paciente com beta-bloqueadores nos primeiros 2 a 3 meses. Clinicamente, há uma clara redução ou eliminação do tom parassimpático com predomínio do simpático. Entretanto há um reequilibrio autonômico natural com redução gradual 
do impulso simpático, levando o ritmo cardíaco aos valores normais. Nesse sentido, há um resultado benéfico de uma longa redução natural do tom simpático (Fig. 17a).

O paciente apresentado na Fig. 15 teve uma frequência cardíaca basal de 62 ppm no teste de inclinação pré-CNA, 85 ppm no controle de 4 meses e 80 ppm no teste de inclinação um ano após a CNA. No entanto, devido ao reajuste do sistema nervoso autônomo, os pacientes geralmente permanecem assintomáticos com a vida normal. Devido a essa plasticidade autonômica, a resposta cronotrópica e a capacidade de exercício também é totalmente preservada, como pode ser visto no estudo do teste de exercício pós-CNA, no qual não foi observada incompetência crônica adicional (Fig. 17b). Um dos problemas que deve ser questionado é se a redução da variabilidade da $R R$ pode aumentar o risco cardiovascular. Esse efeito foi demonstrado após infarto do miocárdio e dano miocárdico, mas não há evidências de que a redução primária da variabilidade $\mathrm{RR}$ causada pela denervação vagal com miocárdio totalmente preservado tenha alguma consequência a esse respeito.

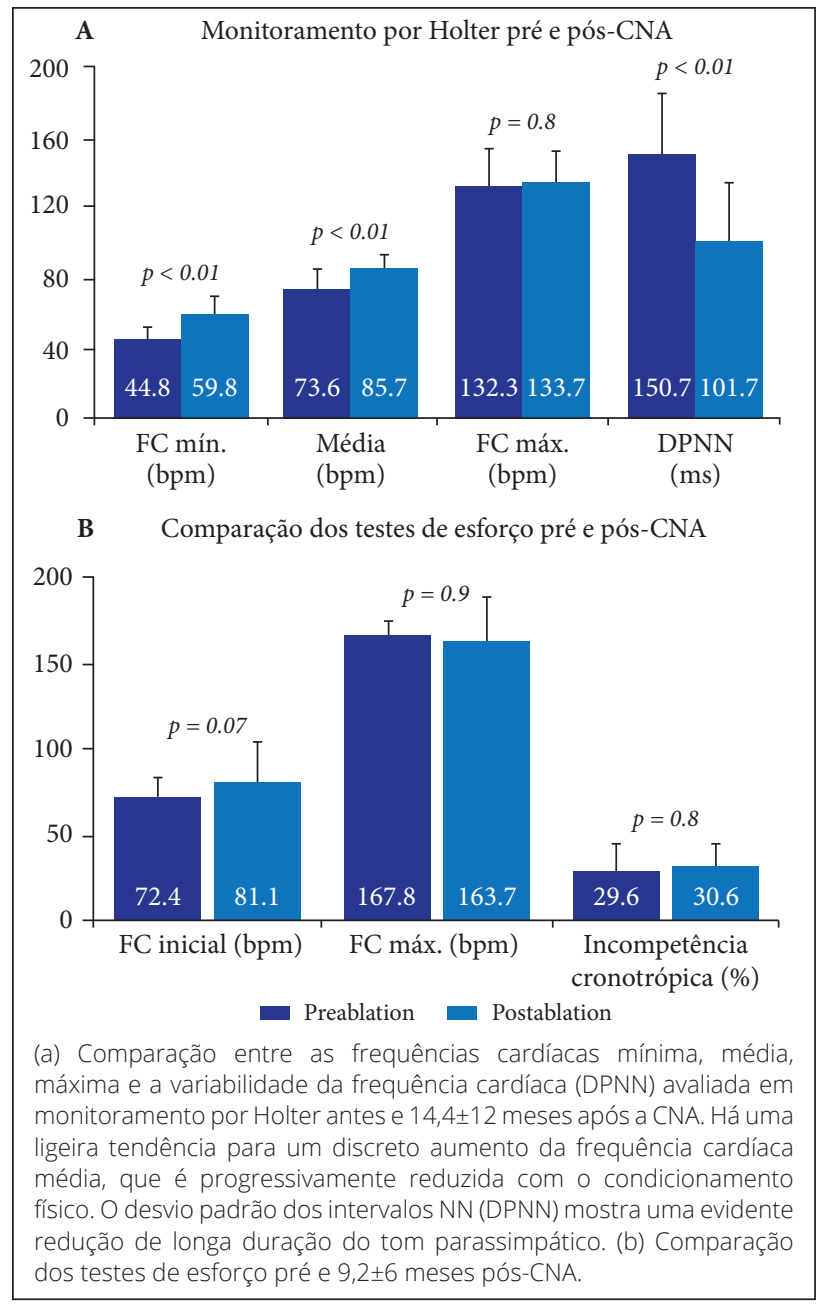

Figura 17. Holter pré e pós-RF.

\section{COMPLICAÇÕES}

Com o cuidado padrão, a CNA tem sido um procedimento muito seguro. A sua taxa de complicações é equivalente à ablação da $\mathrm{FA}$ paroxística em pacientes sem doença cardíaca. Em um estudo de 44 CNA realizadas em 43 pacientes $^{8}$, não foram observadas complicações maiores, apenas dois pequenos hematomas na virilha resolvidos clinicamente por compressão. Um paciente teve um episódio de flutter atrial típico durante a internação hospitalar, revertido por amiodarona intravenosa, não necessitando de terapia a longo prazo. Dez pacientes $(23,3 \%)$ foram temporariamente (1-3 meses) tratados com beta-bloqueadores visando o controle da taquicardia sinusal leve pós-CNA. O condicionamento físico também foi indicado. Não foi necessário implante de marcapasso. Os pacientes tiveram alta após 2 dias.

\section{LIMITAÇÕES}

A CNA está sendo reproduzida por vários estudiosos em todo o mundo com bons resultados semelhantes. No entanto, apesar dos resultados positivos, são necessários ensaios aleatórios para avaliar melhor essa opção terapêutica. A aplicação de RF anatomicamente orientada sobre os PGs ainda não está bem definida e deve ser significativamente melhorada no futuro, especialmente com a inclusão de métodos de imagem, como a ressonância magnética, e/ ou um marcador de gânglios, por exemplo, uma metaiodobenzil-guanidina, que poderia indicar com precisão a posição dos $\mathrm{PGs}$ que podem existir em cada caso. O efeito placebo é comumente observado em todas as alternativas de tratamento para síncope reflexa neuralmente mediada, tais como drogas, implante de marcapasso, educação e treinamento. Entretanto, as mudanças autonômicas significativas e persistentes observadas após a CNA, a abolição da cardioinibição no teste de inclinação de controle e o indubitavelmente melhor resultado a longo prazo, comparado com outras terapias, mostram que o efeito placebo desempenha um papel sem importância nesta terapia (Figura 15). Uma última limitação é que a CNA é um procedimento dependente do operador com resultado altamente relacionado com a curva de aprendizagem. Assim, ensaios randomizados multicêntricos controlados devem esperar pelo treinamento apropriado de vários serviços. 


\section{CONCLUSÃO}

O tratamento da síncope grave cardioinibitória por ablação sem necessidade de marcapasso é muito atrativo, especialmente em pacientes jovens, nos quais a prótese é altamente indesejável. $\mathrm{Na}$ coorte estudada, o marcapasso não foi necessário em nenhum dos casos. Apesar de não prevenir a vasodepressão, a CNA parece causar atenuação do reflexo vagal a longo prazo, eliminando a cardioinibição, e mantendo a maioria dos pacientes assintomáticos. Ao utilizar a atual tecnologia de ablação por fibrilação atrial, o procedimento é seguro, viável e reprodutível. A indicação é simples e essencialmente baseada em constatações clínicas, na presença de cardioinibição grave não responsiva ao tratamento médico, e na resposta normal à atropina. $\mathrm{O}$ estímulo vagal para controlar a extensão da denervação, além de confirmar o sucesso imediato, parece ser decisivo e indispensável. Finalmente, como regra para qualquer nova terapia, os resultados devem ser confirmados com protocolos experimentais e por estudos randomizados.

\section{CONTRIBUIÇÃO DOS AUTORES}

Conceitualização, Mateos José CP; Metodologia, Mateos José CP e Mateos EIP; Redação - primeira versão, Mateos José CP; Redação - Revisão e Edição, Mateos José CP, Mateos EIP, Pachón CTC e Lobo TJ; Aquisição, Mateos José CP; Mateos EIP, Pachón CTC, Lobo TJ, Higuti C, Peña TGS, Mateos Juan CP, Acosta JCZ, Ortencio F e Amarante R; Recursos, Mateos José CP eMateos EIP; Supervisão, Mateos José CP.

\section{REFERÊNCIAS}

1. Sheldon RS, Grubb BP, Olshansky B, Shen WK, Calkins $\mathrm{H}$, Brignole $\mathrm{M}$, et al. 2015 Heart rhythm society expert consensus statement on the diagnosis and treatment of postural tachycardia syndrome, inappropriate sinus tachycardia, and vasovagal syncope. Heart Rhythm. 2015;12(6):e41-63. https://doi.org/10.1016/j. hrthm.2015.03.029

2. Moya A, Sutton R, Ammirati F, Blanc J-J, Brignole M, Dahm JB, et al. Guidelines for the diagnosis and management of syncope (version 2009): The Task Force for the Diagnosis and Management of Syncope of the European Society of Cardiology (ESC). Eur Heart J. 2009;30(21):2631-71. https://doi.org/10.1093/eurheartj/ehp298

3. Brignole M, Menozzi C, Moya A, Andresen D, Blanc J-J, Krahn $A D$, et al. Pacemaker therapy in patients with neurally mediated syncope and documented asystole. Third International Study on Syncope of Uncertain Etiology (ISSUE 3). A randomized trial. Circulation. 2012;125(21):2566-71. https://doi.org/10.1161/CIRCULATIONAHA.111.082313

4. Epstein $A E$, DiMarco JP, Ellenbogen KA, Estes NAM, Freedman RA, Gettes LS, et a. ACC/AHA/HRS 2008 guidelines for device-based therapy of cardiac rhythm abnormalities: Executive summary - A report of the American College of Cardiology/American Heart Association Task Force on Practice Guidelines (Writing Committee to Revise the ACC/AHA/NASPE 2002 Guideline Update for Implantation of Cardiac Pacemakers and Antiarrhythmia Devices). Circulation. 2008;117:2820-40. https://doi.org/10.1161/CIRCUALTIONAHA.108.189741

5. Pachon MJC, Pachon MEI, Pachon MJC, Lobo TJ, Pachon $M Z$, Vargas RN, et al. "Cardioneuroablation" - new treatment for neurocardiogenic syncope, functional AV block and sinus dysfunction using catheter RF-ablation. Europace. 2005;7(1):1-13. https://doi.org/10.1016/j. eupc.2004.10.003

6. Morillo CA, Eckberg DL, Ellenbogen KA, Beightol LA, Hoag JB, Tahvanainen KU, et al. Vagal and sympathetic mechanisms in patients with orthostatic vasovagal syncope. Circulation. 1997;96(8):2509-13. https://doi. org/10.1161/01.cir.96.8.2509

7. Armour JA. Potential clinical relevance of the 'little brain' on the mammalian heart. Exp Physiol. 2008;93(2):16576. https://doi.org/10.1113/expphysiol.2007.041178

8. Pachón MJC, Pachón MEI, Pachón MZC, Lobo TJ, Pachón MJC, Santillana PTG. Catheter ablation of severe neurally meditated reflex (neurocardiogenic or vasovagal) syncope: cardioneuroablation long-term results. Europace. 2011;13(9):1231-42. https://doi.org/10.1093/ europace/eur163

9. Taylor EW, Jordan D, Coote JH. Central control of the cardiovascular and respiratory systems and their interactions in vertebrates. Physiol Rev. 1999;79(3):855916. https://doi.org/10.1152/physrev.1999.79.3.855

10. Santini M, Ammirati F, Colivicchi F, Gentilucci G, Guido $\checkmark$. The effect of atropine in vasovagal syncope induced by head-up tilt testing. Eur Heart J. 1999;20(23):1745-51. https://doi.org/10.1053/euhj.1999.1697

11. Gallego JC, Cubero JS, Pulpón LA, Rodríguez MA, Salas C, Ortiz-Berrocal J. Angina pectoris in the heart transplant recipient: evidence of reinnervation. Rev Esp 
Cardiol. 2001;54(6):799-802. https://doi.org/10.1016/s03008932(01)76396-8

12. Murphy DA, Thompson GW, Ardell JL, McCraty R, Stevenson RS, Sangalang VE, et al. The heart reinnervates after transplantation. Ann Thorac Surg. 2000;69(6):176981. https://doi.org/10.1016/s0003-4975(00)01240-6

13. Coyer BH, Pryor R, Kirsch WM, Blount Júnior SG. Left stellectomy in the long QT syndrome. Chest. 1978;74(5):5846. https://doi.org/10.1378/chest.74.5.584

14. Pappone C, Santinelli V, Manguso F, Vicedomini G, Gugliotta $F$, Augello $G$, et al. Pulmonary vein denervation enhances long-term benefit after circumferential ablation for paroxysmal atrial fibrillation. Circulation. 2004;109(3):32734. https://doi.org/10.1161/01.CIR.0000112641.16340.C7

15. Po SS, Nakagawa H, Jackman WM. Localization of left atrial ganglionated plexi in patients with atrial fibrillation. J Cardiovasc Electrophysiol. 2009;20(10):1186-9. https:// doi.org/10.1111/j.1540-8167.2009.01515.x

16. Driessen AH, Berger WR, Krul SP, van den Berg NW, Neefs J, Piersma FR, et al. Ganglion plexus ablation in advanced atrial fibrillation: The AFACT study. J Am Coll Cardiol. 2016;68(11):1155-65. https://doi.org/10.1016/j. jacc.2016.06.036

17. Aksu T, Baysal E, Guler TE, Yalin K. Selective right atrial cardioneuroablation in functional atrioventricular block. Europace. 2016;19(2):333. https://doi.org/10.1093/ europace/euv413

18. Aksu T, Golcuk E, Yalin K, Guler TE, Erden I. Simplified cardioneuroablation in the treatment of reflex syncope, functional AV block, and sinus node dysfunction. Pacing Clin Electrophysiol. 2016;39(1):42-53. https://doi. org/10.1111/pace.12756

19. Rivarola E, Hardy C, Sosa E, Hachul D, Furlan V, Raimundi $F$, et al. Selective atrial vagal denervation guided by spectral mapping to treat advanced atrioventricular block. Europace. 2016;18(3):445-9. https://doi. org/10.1093/europace/euv142

20. Klank-Szafran M, Stec S, Sledz J, Janion M. Radiofrequency ablation and cardioneuroablation for AVNRT and atrioventricular block. Kardiol Pol. 2010;68(6):720-4.

21. Yao $Y$, Shi R, Wong $T$, Zheng $L$, Chen $W$, Yang $L$, et al. Endocardial autonomic denervation of the left atrium to treat vasovagal syncope: an early experience in humans. Circ Arrhythm Electrophysiol. 2012;5(2):279-86. https:// doi.org/10.1161/CIRCEP.111.966465

22. Liang Z, Jiayou Z, Zonggui W, Dening L. Selective atrial vagal denervation guided by evoked vagal reflex to treat refractory vasovagal syncope. Pacing Clin Electrophysiol. 2012;35(7):e2148. https://doi.org/10.1111/j.1540-8159.2011.03320.x

23. Scanavacca M, Hachul D, Pisani C, Sosa E. Selective vagal denervation of the sinus and atrioventricular nodes, guided by vagal reflexes induced by high frequency stimulation, to treat refractory neurally mediated syncope. J Cardiovasc Electrophysiol. 2009;20(5):558-63. https://doi.org/10.1111/j.1540-8167.2008.01385.x

24. Stavrakis S, Scherlag BJ, Po SS. Autonomic modulation: an emerging paradigm for the treatment of cardiovascular diseases. Circ Arrhythm Electrophysiol. 2012;5(2):247-8. https://doi.org/10.1161/CIRCEP.112.972307

25. Fukunaga $M$, Wichterle $D$, Peichl $P$, Aldhoon B, Cihák $R$, Kautzner J. Differential effect of ganglionic plexi ablation in a patient with neutrally mediated syncope and intermittent atrioventricular block. Europace. 2016;19(1):119-26. https://doi.org/10.1093/europace/ euw100

26. Pachón MJC, Pachón MEl, Pachón MJC, Lobo TJ, Pachón MZ, Vargas RNA, et al. A new treatment for atrial fibrillation based on spectral analysis to guide the catheter RF-ablation. Europace. 2004;6(6):590-601. https://doi.org/10.1016/j.eupc.2004.08.005

27. Chang HY, Lo LW, Lin YJ, Lee SH, Chiou CW, Chen SA. Relationship between intrinsic cardiac autonomic ganglionated plexi and the atrial fibrillation nest. Circ J. 2014;78(4):922-8. https://doi.org/10.1253/circj.cj-131053

28. Singhal R, Lo LW, Lin YJ, Chang SL, Hu YF, Chao TF, et al. Intrinsic cardiac autonomic ganglionated plexi within epicardial fats modulate the atrial substrate remodeling: experiences with atrial fibrillation patients receiving catheter ablation. Acta Cardiol Sin. 2016;32(2):174-84. https://doi.org/10.6515/acs20150427a

29. Oh S, Kong HJ, Choi EK, Kim HC, Choi YS. Complex fractionated electrograms and AF nests in vagally mediated atrial fibrillation. Pacing Clin Electrophysiol. 2010;33(12):1497-503. https://doi.org/10.1111/j.15408159.2010.02834.x

30. Pachon M JC, Arruda M. Spectral mapping identifies critical atrial myocardial sites prone to "electrical resonance": could it be the missing puzzle piece in the physiopathology of AF maintenance? J Cardiovasc Electrophysiol. 2012;23(11):11634. https://doi.org/10.1111/j.1540-8167.2012.02444.x

31. Mateos JCP, Mateos EIP, Lobo TJ, Pachón MZC, Mateos JCP, Pachón DQ, et al. Radiofrequency catheter ablation of atrial fibrillation guided by spectral mapping of atrial fibrillation nests in sinus rhythm. Arq Bras Cardiol. 2007;89(3):124-34/140-50. https://doi.org/10.1590/ s0066-782x2007001500001

32. Arruda $M$, Natale $A$. Ablation of permanent AF: adjunctive strategies to pulmonary veins isolation: targeting AF NEST in sinus rhythm and CFAE in AF. J Interv Card Electrophysiol. 2008;23(1):51-7. https://doi. org/10.1007/s10840-008-9252-z

33. Pachon MJC, Pachon MEI, Santillana PTG, Lobo TJ, Pachon CTC, Pachon MJC, et al. Simplified method for vagal effect evaluation in cardiac ablation and electrophysiological procedures. JACC Clin Electrophysiol. 2015;1(5):451-60. https://doi.org/10.1016/j.jacep.2015.06.008 
34. Pauza DH, Skripka V, Pauziene N, Stropus R. Anatomical study of the neural ganglionated plexus in the canine right atrium: implications for selective denervation and electrophysiology of the sinoatrial node in dog. Anat Rec. 1999;255(3):271-94. https://doi.org/10.1002/ (SICI)1 097-0185(19990701)255:3\%3C271::AID AR4\%3E3.0.CO;2-2

35. Pachon MJC, Pachon MEI, Lobo TJ, Pachon MJC, Pachon $M Z$, Vargas RN, et al. Syncopal high-degree AV block treated with catheter RF ablation without pacemaker implantation. Pacing Clin Electrophysiol. 2006;29(3):31822. https://doi.org/10.1111/j.1540-8159.2006.00340.x

36. Pauza DH, Skripka V, Pauziene N, Stropus R. Morphology, distribution, and variability of the epicardiac neural ganglionated subplexuses in the human heart. Anat Rec. 2000;259(4):353-82. https://doi. org/10.1002/1097-0185(20000801)259:4\%3C353::AIDAR10\%3E3.0.CO;2-R

37. Chiou C-W, Eble JN, Zipes DP. Efferent Vagal Innervation of the Canine Atria and Sinus and Atrioventricular Nodes - The Third Fat Pad. Circulation. 1997;95(11):2573-84. https://doi.org/10.1161/01.cir.95.11.2573

38. Lellouche N, Buch E, Celigoj A, Siegerman C, Cesario D, Diego C. et al. Functional characterization of atrial electrograms in sinus rhythm delineates sites of parasympathetic innervation in patients with paroxysmal atrial fibrillation. J Am Coll Cardiol. 2007;50(14):1324-31. https://doi.org/10.1016/j.jacc.2007.03.069

39. Hachul D. Does non-pharmacological treatment affect outcomes in dysautonomic syndromes? Cardiol J. 2014;21(6):611-5. https://doi.org/10.5603/ CJ.2014.0093

40. Mateos JCP, Mateos EIP, Peña TG, Lobo TJ, Mateos JCP, Vargas RN, et al. Simplified method for esophagus protection during radiofrequency catheter ablation of atrial fibrillation--prospective study of 704 cases. Rev Bras Cir Cardiovasc. 2015;30(2):139-47. https://doi. org/10.5935/1678-9741.20150009

41. Olshansky B, Sullivan RM. Syncope in Patients with Organic Heart Disease. Cardiol Clin. 2015;33(3):449-63. https://doi.org/10.1016/j.ccl.2015.04.013

42. Pauza DH, Skripka V, Pauziene N. Morphology of the intrinsic cardiac nervous system in the dog: a whole-mount study employing histochemical staining with acetylcholinesterase. Cells Tissues Organs. 2002;172(4):297-320. https://doi. org/10.1159/000067198 